RHIC-PH-11

Dilepton Emission and the QCD Phase Transition in Ultrarelativistic Nuclear Collisions

K. Kajantie, J. Kapusta, L. McLerran, A. Mekjian

Brookhaven National Laboratory

June 1986 


\title{
DILEPTON EMISSION AND THE QCD PHASE TRANSITION IN ULTRARELATIVISTIC NUCLEAR COLLISIONS
}

\author{
K. Kajantie \\ Department of Theoretical Physics \\ University of Helsinki \\ Siltavuorenpenger $20 \mathrm{C}$ \\ SF-00170 Helsinki 17 \\ Finland
}

J. Kapusta
School of Physics and Astronomy
University of Minnesota
Minneapolis, Minnesota 55455

L. McLerran

Fermilab

P.O. Box 500

Batavia, IIIinois 60510

\author{
A. Mekjian \\ Department of Physics \\ Rutgers University \\ Piscataway, New Jersey 00854
}

\begin{abstract}
The emission of dileptons from a matter distribution undergoing longitudinal hydrodynamic expansion as might be produced in ultrarelativistic nuclear collisions is computed. Contributions come from quark-gluon plasma, from pion gas and from a mixed phase while the matter undergoes a first order confinement phase transition. This yield is compared with that expected from other sources. It is found that the signals from quark-gluon plasma and pion gas probably exceed such background for sufficiently high initial temperature. The ratio of dilepton rapidity density to the square of the pion rapidity density may provide a measurement of the phase transition temperature. The dilepton mass distribution for various values of transverse energy may provide a measure of vector meson masses as a function of temperature, and a mass shift due to the restoration of chiral symmetry might be seen.
\end{abstract}




\section{Introduction}

In ultrarelativistic nuclear collisions high energy density matter might be produced which would live long enough to be regarded as a system in local thermal equilibrium. If so, a new phase of matter, a quark-gluon plasma, might be made. Various signals have been proposed which may allow a probe of the properties of this new phase. In particular, photons and dileptons may in principle measure properties of this plasma in a relatively clean way since such electromagnetically interacting particles only weakly interact with the hadronic matter in which they are produced. ${ }^{1-6}$ If the dynamics of production of these photons and dileptons is understood then the properties of the matter in which they are produced may be inferred.

In the last year much progress has been made in understanding the dynamics of the expanding matter from which the hadrons are produced 7,8 and in how photons and dileptons are emitted from such expanding matter. ${ }^{9,10}$ Recent hydrodynamic studies indicate that the matter, at least in a fraction of all events, becomes thermalized at some early time, perhaps in the range of . I fm/c<t< $1 \mathrm{fm} / c$, and expands from a quark-gluon plasma, through a mixed phase, and finally a hadron gas. The system probably becomes sufficiently dilute that it goes out of local thermal equilibrium at very late times, $20<t<200 \mathrm{fm} / \mathrm{c}$. The maximum temperature achieved is perhaps $200 \mathrm{MeV}<\mathrm{T}<800 \mathrm{MeV}$, and the temperature at which the system decouples might be $50 \mathrm{MeV}<\mathrm{T}<150 \mathrm{MeV}$. During the time the system exists as a quark-gluon plasma and as a mixed phase, for head on collisions of large nuclei such as gold, the expansion is essentially $1+1$ dimensional ${ }^{7}$, and becomes fully $3+1$ dimensional ${ }^{11}$ only at times of order $t>30 \mathrm{fm} / c$ when the mixed phase has been fully converted to a hadron gas. Of course there may be some thermal gradients induced in the system as it converts from a mixed phase to hadron 
gas since there may be large scale density fluctuations associated with the mixed phase, but given the small expansion rate at this time, we expect that on the average these gradients will be largely washed out. Thus it might be a fair approximation to treat the system as an expanding perfect fluid in most circumstances for most of the lifetime of the system.

To fully treat the emission of photons and dileptons from an expanding quark-gluon plasma it would be useful to employ a full $3+1$ dimensional hydrodynamic simulation of the expansion. Work is in progress on this problem. $^{12}$ To gain semi-quantitative insight, which is perhaps quantitatively valid in the early stages of the expansion, it is sufficient to treat the system as a $1+1$ dimensional system. Such an approximation will have at least two qualitative differences from a more thorough analysis. At late. times the system is not expanding transversely as a fully $3+1$ dimensional treatment would give. During transverse expansion, the matter flows outward, and transverse momentum of collective flow is generated. Moreover, the matter expands more quickly in the $3+1$ dimensional case than in the $1+1$ dimensional case. The treatment of the expansion as $1+1$ dimensional therefore certainiy overestimates the rate of production of low mass and low transverse momentum pairs since these pairs are largely produced from the relatively cold matter late in the collision. Also the higher transverse momentum pairs will be somewhat enhanced due to the collective transverse flow of matter.

Given the history of the expansion of matter produced in an ultrarelativistic collision the rate of emission of dileptons may be computed if the rate is known at each temperature $T$. At high temperatures this rate might be computed in perturbative QCD. However such a computation is plagued by a poorly behaved perturbation expansion even in simple processes such as 
Drell-Yan dilepton emission. Even for very large mass pairs the rate predicted by lowest order perturbation theory and that measured, or computed by summing higher order graphs, are a factor of 2-3 different. ${ }^{13,14}$ Since no serious attempt to sum relevant higher order contributions for a quark-gluon plasma has been attempted we expect perhaps such an uncertainty for even very large mass pairs emitted at very high temperatures. At temperatures near the critical temperature, and for low mass pairs, or for pair masses close to the masses of hadronic resonances, the emission rate is more poorly known. One quantitatively sound way to reliably compute such a rate is a lattice MonteCarlo simulation of the electromagnetic current-current correlation function. Lacking such a computation we must acknowledge that the overall rate which we compute for emission from the plasma is probably not accurate to better than a factor of 2-3, and mass distributions may be even more uncertain near resonant enhancements.

The computation in the hadron gas phase also suffers from numerous inadequacies which may only be properly dealt with by a computation of the electromagnetic current-current correlation function. At temperatures near the critical temperature hadrons overlap and interactions of unknown strength and nature are important. A large number of resonances are also important. The approximate treatment which we present here assumes that the hadron gas consists only of pions. The dileptons are produced by pion annihilation.

The pion is given a form factor which allows for a resonant enhancement due to the rho and rho prime mesons but does not include the effect of other resonances such as the omega. In addition to the obvious inadequacies of such a treatment near the transition temperature this treatment is also inadequate for very low mass pairs $M \leq 2 \pi_{\pi}$. For this mass range the dominant production mechanism for dileptons must necessarily be the bremsstrahlung of a virtual 
photon produced in a pion-pion interaction. At higher masses this contribution is suppressed relative to the annihilation diagram since the bremsstrahlung rate is proportional to the rate of pion-pion interactions with momentum transfer larger than the scale set by the dilepton mass and transverse momentum. Assuming that the system is a dilute, almost ideal, gas of pions requires that the intrinsic rate associated with this process is smaller than the annihilation rate. A computation of the rate of bremsstrahlung emission of photons and dileptons is now in progress. Such a computation, which proper'ly handles the cascading of pions in the hadronic gas phase, will quantify the bremsstrahlung contribution. For small mass pairs the effect of bremsstrahlung is probably of the same order of magnitude as the annihilation contribution.

Given such uncertainties in the theoretical treatment of dilepton emission we should ask if anything semi-quantitative or qualitative might be learned from a treatment as crude as that which we shall present. What we shall argue is that such a computation gives a good semi-quantitative measure of the total emission rate of dileptons of intermediate mass, $0.5 \mathrm{GeV}<\mathrm{M}<4 \mathrm{GeV}$. This gives a fair idea of the magnitude of a signal expected from a thermal distribution of matter as it might be produced in an ultrarelativistic heavy ion collision. Such a treatment also points out qualitatively distinct features of the emission pattern which, on general theoretical grounds, may allow for a measurement of the confinementdeconfinement temperature. It may also allow one to establish that such systems undergo a chiral symmetry restoration phase transition. ${ }^{15-17}$ Finally such computations as we present also establish a general theoretical framework in which theoretically sounder computations may be embedded. 


\section{Expansion Dynamics}

Nuclei at very high energy are expected to be rather transparent to each other. Since we are here only interested in the central rapidity region we shall use Bjorken's longitudinal hydrodynamic model for the space-time evolution of the produced matter. ${ }^{7}$ This model is consistent with the insideoutside cascading observed in proton-nucleus collisions and is expected to be a reasonable first approximation to nucleus-nucleus collisions. In a related paper the effects of allowing transverse expansion are investigated. ${ }^{12}$ It turns out, not surprisingly, that only the late stage of the reaction is significantly affected, not the early high temperature quark-gluon plasma epoch.

The important features of the longitudinal hydrodynamic model are:

(1) The produced matter is baryon free and electrically neutral.

(2) Rapidity distributions are invariant under velocity boosts along the beam axis. (3) The thermalized matter is produced a proper time $t_{0}$ after nuclear impact with initial entropy density $s_{0}$. Thereafter the entropy density falls according to

$$
s(t)=s_{0} t_{0} / t
$$

The thermal emission rate of dileptons depends on the temperature $T$ (see sect. III). Therefore we need to know the relationship between $s$ and $T$, i.e., the equation of state.

Our pragmatic point of view in this paper is to describe the quark-gluon plasma as a noninteracting gas of eight massless gluons and two flavors ( $u, d$ ) of massless quarks. (With a little extra work strange quarks could also be included, and it will èventually be necessary to do so when comparing with 
experiment. However in this paper we consistently neglect strangeness for simplicity.) A bag constant $B$ is used to simulate the effects of confinement. The hadron phase is described as a noninteracting gas of massless pions. This should be a fair approximation for temperatures in the range $100 \mathrm{MeV}<\mathrm{T} 240 \mathrm{MeV}$, adequate for our purposes. Thus the pressure, energy - density and entropy density in each of the two phases are

$$
\begin{aligned}
& P_{q}=37 a T^{4}-B \\
& \varepsilon_{q}=111 a T^{4}+B \\
& s_{q}=148 a T^{3} \\
& P_{h}=3 a T^{4} \\
& \varepsilon_{h}=9 a T^{4} \\
& s_{h}=12 a T^{3} \\
& a=\pi^{2} / 90 .
\end{aligned}
$$

The critical temperature is determined by pressure balance to be

$$
T_{C}=(B / 34 a)^{1 / 4} \text {. }
$$

Thus $\mathrm{B}$ may be eliminated in favor of $\mathrm{T}_{C}$. The latent heat necessary to liberate the color degrees of freedom is $4 \mathrm{~B}$.

At $T_{C}$ it is further necessary to specify the volume fraction $f$ of the quark-gluon phase. The entropy density is

$$
s\left(f, T_{c}\right)=s_{q}\left(T_{c}\right) f+s_{h}\left(T_{c}\right)(1-f)
$$

and similarly for the energy density. 
Assume now that the nucleus-nucleus collision produces quark-gluon plasma with initial entropy density $s_{0}>s_{q}\left(T_{c}\right)$. Following eqs. (2.1) and (2.2) the system expands adiabatically in the quark-gluon phase according to

$$
T(t)=T_{0}\left(t_{0} / t\right)^{1 / 3}
$$

until $T$ drops to $T_{c}$. This occurs in the proper time interval $t_{0}<t \leq t_{1}=$ $\left(\mathrm{T}_{0} / \mathrm{T}_{\mathrm{c}}\right)^{3} \mathrm{t}_{0^{\circ}}$. Assuming that nucleation of the hadron phase is fast the system then enters the mixed phase. In the mixed phase the entropy density decreases not by decreasing $\mathrm{T}$ but by converting quark-gluon plasma to hadron matter at lower entropy density but still at $T_{c}$. The fraction $f(t)$ is determined by eqs. (2.1) - (2.4) to be

$$
f(t)=\frac{1}{r-1}\left(r \frac{t_{1}}{t}-1\right)
$$

where $r=37 / 3$ is the ratio of the number of degrees of freedom in the two phases. Thus $1>f>0$ for $t_{1}<t<t_{2}=r t_{1}$. The mixed phase terminates at $t_{2}$ whereupon the temperature begins to fall again according to

$$
T(t)=T_{c}\left(t_{2} / t\right)^{1 / 3}
$$

for $t>t_{2}$. The expansion continues until the pions can no longer maintain thermal contact. We take this as a final break-up temperature $\mathrm{T}_{f}$. In the future it should be possible to avoid this sharp cut-off by implementing a pion cascade code. ${ }^{18}$ See Fig. 1. 
In case $s_{q}\left(T_{c}\right)>s_{0}>s_{h}\left(T_{c}\right)$ we assume that the matter is initially formed in the mixed phase with volume fraction $f_{0}$ determined by

$$
s_{0}=s_{q}\left(T_{c}\right) f_{0}+s_{h}\left(T_{c}\right)\left(1-f_{0}\right)
$$

It follows that

$$
f(t)=\frac{1}{r-1}\left[\left(1+(r-1) f_{0}\right) \frac{t_{0}}{t}-1\right]
$$

The system evolves in the mixed phase until time $t_{2}=\left(1+(r-1) f_{0}\right) t_{0}$. Subsequently it follows eq. (2.7) in the hadron phase.

Finally, if no quark-gluon plasma is formed at all but only hadron matter, then the temperature follows eq. (2.5) until the time of thermal. break-up.

An interesting and useful feature of Bjorken's model is that the entropy per unit of rapidity is conserved during the expansion. It is

$$
\mathrm{dS} / \mathrm{dy}=\operatorname{area} \cdot \mathrm{s}(\mathrm{t}) \mathrm{t}=\text { constant }
$$

where the cross-sectional area of the produced matter enters. Since the entropy density of a gas of massless pions is proportional to the pion number density it follows that the entropy can be determined by measuring the charged particle multiplicity. ${ }^{10}$ Considering a head-on collision of equal mass nuclei one finds

$$
\frac{d N_{c h}}{d y}=\left[f_{0}+\frac{1-f_{0}}{r}\right] 3 \pi R^{2} t_{0} T_{0}^{3},
$$


where $R$ is the nuclear radius, $f_{0}=0$ if $T_{0}<T_{c}, 0 \leq f_{0} \leq 1$ if $T_{0}=T_{c}$, and $f_{0}=1$ if $T_{0}>T_{c}$.

The above discussion is not significantly altered even if the rather large latent heat is shrunk to zero so that the first order phase transition turns into a second order one, or even if there is no proper phase transition. at a11. The essential requirement is that the number of degrees of freedom increase by a factor of $\mathrm{r}$ in a small temperature interval $\Delta \mathrm{T}$.

\section{Emission Rates}

\section{A. Cross Sections}

The production of dileptons from a thermal system at temperature $T$ will now be considered. In this paper only the processes

$$
a^{+}+a^{-} \rightarrow l^{+}+l^{-}
$$

will be included, where ' $a$ ' is a pion or a quark. Our estimates will be based on kinetic theory. Thus the cross section for (3.1) must be known. From QED one can calculate the cross section for $e^{+} e^{-} \rightarrow \mu^{+} \mu^{-}$. It is

$$
\tilde{\sigma}(M)=\frac{4 \pi}{3} \frac{\alpha^{2}}{M^{2}}\left(1+\frac{2 \mathrm{~m}_{\ell}^{2}}{M^{2}}\right)\left(1-\frac{4 \mathrm{~m}_{\ell}^{2}}{\mathrm{M}^{2}}\right)^{1 / 2}
$$

where $\alpha$ is the fine structure constant, $m_{\ell}$ is the muon mass and $M$ is the center of mass energy. Note that this cross section is spin-averaged in the initial state and spin-summed in the final state.

For quark-antiquark annihilation the above formula is modified by a color factor which enhances the cross section by $N_{c}=3$. Also it is modified by a 
factor reflecting the fractional electrical charges of the quarks: u quarks have charge $2 / 3$, d quarks have charge $-1 / 3$, etc. Thus the cross section for $\ell^{+} \ell^{-}$production by quarks is

$$
\begin{gathered}
\sigma_{q}(M)=F_{q} \tilde{\sigma}(M), \\
F_{q}=N_{c}(2 s+1)^{2} \sum_{f} e_{f}^{2},
\end{gathered}
$$

where the sum is over the different quark flavors. For $u$ and $d$ quarks, which is all we will include in our numerical analysis, $\mathrm{F}_{\mathrm{q}}=20 / 3$. If massless $\mathrm{s}$ quarks were also to be included then $\mathrm{F}_{\mathrm{q}}$ would increase by $20 \%$ to $24 / 3$. Note that (3.3), in contrast to (3.2), is spin-summed in the initial state. The cross section for $\ell^{+} \ell^{-}$production by pions has a different type of modification. The analogy of the spin-color charge weight factor is one for $\pi^{+} \pi^{-} \rightarrow \ell^{+} \ell^{-}$since pions are spinless, colorless and have one unit of electric charge. Vector meson dominance says that the primary channels for the annihilation are

$$
\begin{aligned}
& \pi^{+}+\pi^{-} \rightarrow \rho \rightarrow \ell^{+}+\ell^{-} \\
& \pi^{+}+\pi^{-} \rightarrow \rho^{\prime} \rightarrow \ell^{+}+\ell^{-}
\end{aligned}
$$

Thus we must multiply (3.2) by a form factor, which we shall take as a sum of Breit-Wigner's. 


$$
\begin{gathered}
F_{\pi}(M)=\frac{m_{\rho}^{4}}{\left(m_{\rho}^{2}-M^{2}\right)^{2}+m_{\rho^{2}}^{2} \Gamma_{\rho}^{2}}+\frac{1}{4} \frac{m_{\rho^{\prime}}^{4}}{\left(m_{\rho^{\prime}}^{2}-M^{2}\right)^{2}+m_{\rho^{\prime}}^{2} \Gamma_{\rho^{\prime}}^{2}}, \\
m_{\rho}=775 \mathrm{MeV}, \Gamma_{\rho}=155 \mathrm{MeV} \\
m_{\rho^{\prime}}=1600 \mathrm{MeV}, \Gamma_{\rho^{\prime}}=260 \mathrm{MeV} .
\end{gathered}
$$

The $1 / 4$ follows because the branching ratio for $\rho^{\prime} \rightarrow \pi^{+} \pi^{-}$is $25 \%$ whereas for the $\rho$ it is essentially $100 \%$. Thus the pion cross section is

$$
\sigma_{\pi}(M)=F_{\pi}(M) \tilde{\sigma}(M)\left(1-4 m_{\pi}^{2} / M^{2}\right)^{1 / 2}
$$

Other vector mesons which couple to $\pi^{+} \pi^{-}$are not included. For example, the $\omega$ has a branching ratio into $\pi^{+} \pi^{-}$of only $1.3 \%$ (the dominant mode is into three pions) and the next lowest lying vector meson is the $\rho(2150)$ which is seen only as a very broad resonance in $\bar{p} p \rightarrow \pi^{+} \pi^{-}$. If $s$ quarks were included then the $\phi(1020)$ would be a big contributor, and likewise if $c$ quarks were included it would be the $\psi(3100)$.

Fig. 2 compares $F_{q}$ and $F_{\pi}(M)$. The resonant enhancement is seen to be quite important near the $\rho$ and $\rho$ 'masses.

\section{B. Statics}

Kinetic theory gives the reaction rate (number of such reactions per unit time per unit volume) as 


$$
\begin{gathered}
R\left(a^{+} a^{-} \rightarrow l^{+} l^{-}\right)=\int \frac{d^{3} p_{1}}{(2 \pi)^{3}} f\left(\vec{p}_{1}\right) \int \frac{d^{3} p_{2}}{(2 \pi)^{3}} f\left(\vec{p}_{2}\right) \\
\cdot \sigma\left(a^{+} a^{-} \rightarrow l^{+} l^{-} ; \vec{p}_{1}, \vec{p}_{2}\right) v_{r e 1}, \\
v_{r e 1}=\frac{\sqrt{\left(p_{1}: \dot{p}_{2}\right)^{2}-m_{a}^{4}}}{E_{1} E_{2}} .
\end{gathered}
$$

Here $f(\vec{p})$ is the occupation probability in momentum space. For our specific applications it turns out that quantum effects are not important but relativisitic effects are. Thus we use

$$
\begin{aligned}
f(\vec{p}) & =\exp (-E / T), \\
E^{2} & =\vec{p}^{2}+m_{a}^{2}
\end{aligned}
$$

Pauli blocking of the final state is not important (see below) so that effect is not included in (3.7) either.

Inserting (3.8) into (3.7) and integrating over five of the six variables we find

$$
R(T)=\frac{T^{6}}{(2 \pi)^{4}} \int_{0}^{\infty} \sigma(z) z^{2}\left(z^{2}-4 z_{a}^{2}\right) K_{1}(z) d z,
$$

where $z=M / T, z_{a}=m_{a} / T$ and $z_{0}$ is the larger of $2 m_{a} / T$ and $2 m_{\ell} / T$. For example, considering $e^{+} e^{-}$production by $u$ and $d$ quarks so that all particles may essentially be treated as massless compared with $T$, one finds that 
$R=\frac{10}{9 \pi^{3}} \alpha^{2} T^{4}$. The corresponding relaxation time for electrons to come to chemical equilibrium with the quarks is approximately

$$
t_{r e 1}=n_{e^{e q}}^{e} / 2 R=9 \pi / 10 \alpha^{2} \mathrm{~T}
$$

For $\mathrm{T}=200-500 \mathrm{MeV}, \mathrm{t}_{\mathrm{rel}}=2-6 \times 10^{4} \mathrm{fm} / \mathrm{c}$. This time is much greater than the lifetime of quark-gluon plasma produced in high energy heavy ion collisions. Therefore the produced leptons should be emitted from the system without any appreciable absorption, and so $R$ will be taken to be the rate of emission of lepton pairs.

Apart from the total number of lepton pairs emitted per unit space-time, which is

$$
d N / d^{4} x=R
$$

we will be interested in several differential rates. These may be obtained from (3.7) by an appropriate change of variables. The rate for producing pairs with invariant mass squared $\mathrm{M}^{2}$ is

$$
\frac{d N}{d^{4} x d M^{2}}=\frac{\sigma(M)}{2(2 \pi)^{4}} M^{3} T K_{1}(M / T)\left(1-\frac{4 m_{a}^{2}}{M^{2}}\right)
$$

The rate for producing pairs with $\mathrm{M}^{2}$ and with transverse energy $E_{T}=\left(p_{T}^{2}+M^{2}\right)^{1 / 2}$, where $p_{T}$ is the momentum transverse to the beam axis, is

$$
\frac{d N}{d^{4} x d M^{2} d E_{T}}=\frac{\sigma(M)}{4(2 \pi)^{4}} M^{2} E_{T} K_{0}\left(E_{T} / T\right)\left(1-\frac{4 m_{a}^{2}}{M^{2}}\right) .
$$




\section{Dynamics}

We will now integrate the emission rates found previously over the spacetime volume of the reaction according to Bjorken's model. In this model the volume element is

$$
d^{4} x=d^{2} x_{T} d y \text { tdt }
$$

where $t$ is the proper time and $y$ is the rapidity of the fluid element. In this paper we shall focus on central collisions of equal mass nuclei so the integration over transverse coordinates just yields a factor of $d^{2} x_{T}=\pi R_{A}^{2}$, where $R_{A}$ is the nuclear radius.

First consider the total number of lepton pairs emitted by the quark phase when $\mathrm{T}_{0}>\mathrm{T}>\mathrm{T}_{\mathrm{c}}$ and $\mathrm{t}_{0}<\mathrm{t}<\mathrm{t}_{1}$ (referred to as the cooling line),

$$
\frac{d N^{q}}{d y}=\pi R_{A}^{2} \int_{0}^{t_{1}} d t t R^{q}(T(t))
$$

It is convenient to change the variable of integration from to $T$ with the help of (2.5).

$$
\frac{d N^{q}}{d y}=3 \pi R_{A}^{2} t_{0}^{2} T_{0}^{6} \int_{C}^{T} d T T^{-7} R^{q}(T)
$$

When $m_{\ell}$ can be neglected compared with $T$ an analytical result may be found, 


$$
\frac{d N^{q}}{d y}=\frac{\alpha^{2}}{(2 \pi)^{2}} R_{A}^{2} F_{q} t_{0}^{2} T_{o}^{4}\left(\left(T_{o} / T_{c}\right)^{2}-1\right)
$$

It turns out that this is a fairly good approximation even for muons since $T_{c}>m_{\mu}$

Along the Maxwell coexistence line the temperature is fixed at $T_{c}$ but the rate must be multiplied by the volume fraction $f(t)$ occupied by plasma,

$$
\frac{d N^{q}}{d y}=\pi R_{a}^{2} R^{q}\left(T_{c}\right) \int_{t_{1}^{2}}^{t_{2}} f(t) t d t
$$

where

$$
\int_{1}^{t_{2}} f(t) t d t=\frac{1}{2}(I-1) f_{0}^{2} t_{1}^{2}
$$

This result holds when the system starts in the quark phase $\left(T_{0}>T_{c}, f_{0}=1\right.$, . $\left.t_{1}>t_{0}\right)$ or when the system starts in the mixed phase $\left(T_{0}=T_{c}, 0<f_{0}<1\right.$, $\left.t_{1}=t_{0}\right)$.

In general the contribution from pion annihilation cannot be obtained analytically. Thus, on the cooling line $T<T_{c}$,

$$
\frac{d N^{\pi}}{d y}=3 \pi R_{A}^{2} t_{2}^{2} T_{c}^{6} \int_{T_{f}}^{T} d_{T T}^{c} R^{\pi}(T),
$$

analogous to (3.16). Along the Maxwell coexistence line

$$
\frac{d N^{\pi}}{d y}=\pi R_{A}^{2} R^{\pi}\left(T_{c}\right) \int_{1}^{t_{2}}[1-f(t)] t d t,
$$


where

$$
\int_{t_{1}}^{t_{2}}[1-f(t)] t d t=(r-1)\left[1+\frac{1}{2}(r-2) f_{0}\right] f_{0} t_{1}^{2} .
$$

There still remains the space-time integration for the distributions $\mathrm{dN} / \mathrm{dydM}^{2}$ (3.12) and $\mathrm{dN} / \mathrm{dydM}^{2} \mathrm{dE}_{\mathrm{T}}$ (3.13). Along the Maxwel1 coexistence Iine the relevant integrals have already been given, namely (3.19) and (3.22). On the cooling line in the quark phase one finds

$$
\begin{aligned}
& \int_{0}^{t_{1}} T K_{1}(M / T) t d t=3 T_{0}^{6} t_{0}^{2} M^{-5}\left(H\left(M / T_{0}\right)-H\left(M / T_{c}\right)\right) \\
& H(z)=z^{2}\left(8+z^{2}\right) K_{0}(z)+4 z\left(4+z^{2}\right) K_{1}(z) ;
\end{aligned}
$$

and

$$
\begin{gathered}
\int_{0}^{t_{1}} K_{0}\left(E_{T} / T\right) t d t=3 E_{T}^{-6} T_{0}^{6} t_{0}^{2}\left[G\left(E_{T} / T_{0}\right)-G\left(E_{T} / T_{c}\right)\right] \\
G(z)=z^{3}\left(8+z^{2}\right) K_{3}(z) .
\end{gathered}
$$

On the cooling line in the pion phase the same results apply with the replacements $t_{0} \rightarrow t_{2}, t_{1} \rightarrow t_{f}, T_{0} \rightarrow T_{c}, T_{c} \rightarrow T_{f}$.

This concludes our discussion of the thermal emission rates based on kinetic theory. The distributions $\mathrm{dN} / \mathrm{dydM}^{2}$ and $\mathrm{dN} / \mathrm{dydM}^{2} \mathrm{dE}_{\mathrm{T}}$ can be written down in closed form albeit in terms of the Bessel functions $K_{n}(z)$. Along the Maxwell coexistence Iine also $\mathrm{dN} / \mathrm{dy}$ can be written down in closed form. Along the cooling lines the quark phase contribution can be written down in closed form if the mass of the produced leptons can be neglected, whereas the pion 
phase contribution always requires a one-dimensional numerical integration due to the complicated form factor.

\section{Observed Distributions}

In this section we discuss the dilepton distributions derived in the previous section and which might be observed in central collisions of ${ }^{197} \mathrm{Au}$ nuclei at a center of mass beam energy of 10 to $100 \mathrm{GeV} /$ nucleon. All parameters have previous $1 \mathrm{y}$ been given except that now we specify $t_{0}=1 \mathrm{fm} / \mathrm{c}$, $\pi R_{A}^{2}=127 \mathrm{fm}^{2}$ and $T_{f}=100 \mathrm{MeV}$.

Figure 3 shows the various contributions to the ${\mu^{+}}^{+} \mu^{-}$invariant mass distribution $\mathrm{dN} / \mathrm{dydM}^{2}$ for three combinations of initial and critical. temperatures $T_{0}$ and $T_{C}$. Resonant pion annihilation generally dominates quark annihilation in the mass range $0.4 \mathrm{GeV}<\mathrm{M}<2 \mathrm{GeV}$. This is due to the big peaks in the pion form factor (Fig. 2) and to the relatively long time spent in the pion phase (Fig. 1). (Allowance for transverse expansion would reduce the time spent in the pion phase and so would reduce its contribution.) The quark phase contribution breaks through at about $M=2 \mathrm{GeV}$ due to the fact that quarks annihilate at a higher average temperature than do the pions. Actually the peaks due to the vector mesons can be turned to our advantage. It has been suggested that at least the $\rho$ mass, but possibly the $\rho^{\prime}$ mass also, will decrease as the temperature is increased as a precursor to chiral symmetry restoration. Observation of such a mass shift would be quite exciting in itself. Our calculated distribution in the mass range $M<0.4 \mathrm{GeV}$ is probably not very reliable since dilepton production via virtual bremsstrahlung has not been included.

Also shown for comparison is the low mass spectrum measured in pp collisions ${ }^{19}$ and scaled by $A^{2 / 3}$. This scaling is suggested by observations on 
proton-nucleus collisions: the cross section at low mas scales like $\mathrm{A}^{2 / 3}$, indicative of a highly absorptive (surface or black disk) mechanism. ${ }^{19}$ Then central A + A collisions should also scale like $A^{2 / 3}$ if there was no change in the reaction mechanism. The two peaks correspond to the $\rho$ and the $\phi$. Currently we do not include strange quarks in our calculation so there is no $\phi$ peak in our results. We expect the mechanism for producing low mass dileptons in $\mathrm{Au}+\mathrm{Au}$ collisions to be quite different than in pp collisions. ${ }^{20}$ The reason for plotting the scaled pp spectrum is to illustrate the background if no thermalized matter is formed.

The Drell-Yan ${ }^{21}$ mechanism produces very high mass dileptons by the first hard quark-antiquark annihilations in the incoming nuclei. By the uncertainty principle this is a short time pre-equilibrium phenomenon which must be there. In pp collisions

$$
\frac{d \sigma}{d y d M^{2}}=M^{-4} f(M / \sqrt{s})
$$

When $M \ll \sqrt{ } s$ the function $f$ can be well-approximated by $f(0)$. This is a11 right for the energies and mass range under consideration here. As mentioned earlier the Drell-Yan result typically must be multiplied by a $K$ factor of about 2 to bring agreement with experiment. This factor can be explained by summing higher order QCD diagrams ${ }^{13,14}$. In proton-nucleus collisions it has been observed that high mass pairs scale like ${ }^{19} \mathrm{~d} \sigma / \mathrm{dydM}^{2} \alpha$ A. This is expected on the basis of the Glauber multiple scattering theory if the constituents (very high moment components of the quark and antiquark wavefunctions) are rather transparent. For A + A collisions the cross section 
would scale like $A^{2}$ and for central collisions the scaling would be $A^{4 / 3}$. The latter factor has been used in Fig. 3.

It can be seen that the Drell-Yan contribution is dominant for $M>2 \mathrm{GeV}$ if the initial temperature is relatively low, $T_{0}=250 \mathrm{MeV}$. If the initial temperature is increased to $T_{0}=500 \mathrm{MeV}$ then the thermal quark phase contribution dominates the Drel1-Yan by two orders of magnitude. Note, however, that the Drell-Yan contribution is essentially a known quantity and can be subtracted from the experimental observations.

Next we consider the total number of dileptons emitted per unit rapidity irrespective of whether they come from the quark phase or the pion phase. We recall from sect. III that $\mathrm{dN} / \mathrm{dy}$ involves an integration factor tdt and therefore is proportional to $t_{0}^{2}$. On the other hand the charged pion rapidity density $\mathrm{dN}_{\mathrm{ch}} / \mathrm{dy}$ is proportional to $\mathrm{t}_{\mathrm{o}}$. Thus the ratio $\mathrm{dN} / \mathrm{dy} /\left(\mathrm{dN}_{\mathrm{ch}} / \mathrm{dy}\right)^{2}$ is independent of the numerical value of $t_{0}$. This ratio is plotted as a function of $\mathrm{dN}^{\mathrm{ch}} / \mathrm{dy}$ in Fig. 4. This figure illustrates a number of remarkable properties. The ratio is a monotonically increasing function as long as the thermalized matter appears initially in the pion phase. As the initial energy density is increased, mixed-phase begins to form and the ratio begins to turn over. Once the initial energy density surpasses the threshold to form only quark phase then a plateau is reached! The height of this plateau is independent of the initial conditions, that is, of $t_{0}$ and $T_{0}$, as long as $T_{0}>$ $T_{c}$. Furthermore we find that the height is proportional to $T_{c}$. The reason can be seen in Fig. 1. The system lives at a higher average temperature as $T_{c}$ is increased even though the duration of the expansion is the same. Thus it may be possible eventually to not only verify that quark-gluon plasma was formed but also to infer $\mathrm{T}_{c}$ from experimental observation of the dilepton rapidity density. 
The precise height of the plateau is complicated by several effects outside the scope of the Bjorken model. Allowing for transverse expansion shortens the lifetime of the pion phase (not so much the quark phase) and so would reduce the height. There is also some sensitivity to the final temperature $\mathrm{T}_{f}$ where pions are assumed to lose thermal contact with each other. For example, changing $\mathrm{T}_{f}$ by $10 \%$ also changes the plateau height by about 10\%. Both of these effects could be addressed in a pion cascade code.

In Fig. 5 we plot the dimuon mass distribution for fixed values of the transverse energy. The combinations of $\mathrm{T}_{0}$ and $\mathrm{T}_{C}$ are the same as in Fig. 3 . With no transverse expansion the average transverse energy of a pair increases with the temperature at which the pair was formed. Quark annihilation proceeds at $\mathrm{T} \geq \mathrm{T}_{\mathrm{c}}$ while pion annihilation proceeds at $\mathrm{T} \leq \mathrm{T}_{\mathrm{c}}$. Therefore as $\mathrm{E}_{\mathrm{T}}$ is increased the relative contribution of pion phase decreases as the figure clearly shows. This extinction of vector meson peaks has been suggested as a test for quark gluon plasma formation. 22 The greater the ratio $\mathrm{T}_{\mathrm{o}} / \mathrm{T}_{\mathrm{c}}$ the faster the vector meson peaks decrease with $\mathrm{E}_{\mathrm{T}}$. This phenomenon is, however, dependent on the absence of transverse expansion. In a full $3+1$ dimensional model the transverse flow velocity would increase with time while the temperature would decrease with time. Thus dimuons emitted late in the reaction from the pion phase receive a greater kick which reduces the extinction mechanism. ${ }^{12}$

V. Conclusion

In this work we used Bjorken's model to study dilepton emission in central collisions of massive nuclei at ultrarelativistic energies. The basic results can be summarized as follows. Resonant pion annihilation dominates quark annihilation in the mass range between 0.5 and $2 \mathrm{GeV}$. This seems to be 
true no matter. how high the initial temperature is. It is a consequence of the long emission time of the pion phase compared to the quark phase. However this can be used to advantage since the vector meson masses and widths are expected to be temperature dependent ${ }^{15,17}$ and consequences of chiral symmetry restoration might be seen.

The ratio of the dilepton rapidity density to the square of the pion rapidity density reaches a plateau at high initial temperature, generally when $T_{0}>T_{c}$. The height of the plateau is independent of the two most uncertain parameters in Bjorken's model, namely the thermalization time $t_{0}$ and the initial temperature $T_{0}$. The height is proportional to $T_{c}$. Thus it might be possible to determine the critical temperature experimentally.

The vector meson peaks do diminish in height as-the transverse energy of the pair is increased. This is a direct consequence of pion annihilation proceeding at lower temperature than quark annihilation.

The above conclusions could be altered to some extent by improvements not considered here, which we must be cautious not to underestimate. We mention just five of the most significant ones below.

We used vector meson dominance to estimate the pion electromagnetic form factor. It would be better to use the form factor as measured in $e^{+} e^{-}$ annihilation into $\pi^{+} \pi^{-}$. Especially the $\rho^{\prime}$ peak is not so evident as vector meson dominance implies. More generally multipion annihilation could be important at high energy density but this is difficult to analyze.

In this work we assumed that the nucleation of the hadron phase is fast and that the system goes through a mixed phase at constant pressure and temperature (Maxwell construction). If nucleation is slow more complicated scenarios could ensue, such as supercooling, explosive bubble growth, entropy production, etc. Such scenarios should be considered, but we find it 
advisable to stay with the simplest one at the present state of theoretical development of the dynamics.

A better hydrodynamic treatment of the collision would take into account transverse as well as 1ongitudinal expansion. ${ }^{11}$ This is now being done. ${ }^{12}$ In order to avoid the sharp transition from hydrodynamic flow to free-streaming . (break-up, or final, temperature $\mathrm{T}_{f}$ ) it would be advantageous to have a pion cascade code. Such a project is also underway. 18

So far the calculation of the low mass spectrum, $M<500 \mathrm{MeV}$, is incomplete. The low mass spectrum is sensitive to the quark and to the pion dispersion relations in the high temperature medium. ${ }^{16}$ We have used the simple dispersion relations $\omega=k$ for quarks and $\omega=\left(m_{\pi}^{2}+k^{2}\right)^{1 / 2}$ for pions, but it would be very interesting to repeat these calculations with other possible dispersion relations which take into account interactions among the particles. Also there will be an enhancement of the low mass spectrum coming from virtual bremsstrahlung in pion-pion and quark-quark collisions. These rates have not yet been calculated.

We have considered what background might come from elementary nucleonnucleon collisions, but actually the definition of background is not unique. What we search for is evidence of an extended system living for a long time. Background should somehow refer to the quantum pre-equilibrium processes occurring at times less than the thermalization time $t_{0} \cdot 23,24$ In our calculation these quantum processes determine the initial conditions and it is not clear how background should be separated. One pragmatic way of defining background would be to consider extensions of the standard low $\mathrm{p}_{\mathrm{T}}$ hadron models (multi-chain, dual parton, string, etc.) to nucleus-nucleus collisions. To our knowledge this has not yet been done for dilepton production. 
We conclude that dilepton production remains a promising probe of QCD dynamics in ultra-relativistic nucleus-nucleus collisions.

\section{Acknowledgments}

J. K.'s research was supported by the U. S. Department of Energy under contract DOE/DE-ACO2-79ER-10364, A.M.'s research was supported by the National Science Foundation. 


\section{References}

1. E. L. Feinberg, Nuovo Cimento 34A, 391 (1976).

2. E. V. Shuryak, Phys. Lett. 78B, 150 (1978).

3. G. Domokos and J. I. Goldman, Phys. Rev. D223, 203 (1981); G. Domokos, ibid., 28, 123 (1983).

4. K. Kajantie and H. I. Miettenen, Z. Phys. C9, 341 (1981); C14, 357 (1982).

5. S. Chin, Phys. Lett. 119B, 51 (1982).

6. B. Sinha, Phys. Lett. 128B, 91 (1983); 157B, 221 (1985); and Bhabha Atomic Research Center preprint (1986).

7. J. D. Bjorken, Phys. Rev. D27, 140 (1983).

8. J. Kapusta and A. Mekjian, Phys. Rev. D333, 1304 (1986).

9. L. D. McLerran and T. Toimela, Phys. Rev. D31, 545 (1985).

10. R. C. Hwa and K.'Kajantie, Phys. Rev. D32, 1109 (1985).

11. E. von Gersdorff, M. Kataja, L. McLerran and P: V. Ruuskanen, Fermilab preprint no. 86/13-T (1986).

12. K. Kajantie, M. Rataja, L. McLerran and P. V. Ruuskanen, University of Helsinki preprint no. HU-TFT-86-6 (1986).

13. G. Altarelii, R. K. Ellis and G. Martinelli, Phys. Lett. 151B, 457 (1985).

14. Y. Gabellini, J. L. Meunier and G. Plaut, Z. Phys. C29, 439 (1985).

15. R. D. Pisarski, Phys. Lett. 110B, 155 (1982).

16. J. Kapusta, Phys. Lett. 136B, 201 (1984).

17. A. I. Bochkarev and M. E. Shaposnikov, Phys. Lett. 145B, 276 (1984).

18. G. Bertsch and L. McLerran, to be published.

19. R. Stroynowski, Phys. Rep. 71, 1 (1981).

20. J. D. Bjorken and H. Weisberg, Phys. Rev. D13, 1405 (1976).

21. S. D. Drel1 and T. M. Yan, Phys. Rev. Lett. 25, 316 (1970); Ann. Phys. (NY) 66,578 (1971).

22. P. J. Siemens and S. A. Chin, Phys. Rev. Lett. 55, 1266 (1985). 
23. V. Cerny, P. Lichard and J. Pisut, Phys. Lett. 70B, 61 (1977); Phys. Rev. D16, 2822 (1977); D18, 2409 (1978); D24, 652 (1 $\overline{981}$ ).

24. A. Bialas and J.-P. Blaizot, Phys. Rev. D32, 2954 (1985).

\section{Figure Captions}

Fig. 1 The time evolution of the temperature in Bjorken's model for initial condition $\mathrm{T}_{0}=250 \mathrm{MeV}$ at $t_{0}=1 \mathrm{fm} / \mathrm{c}$. Two different values of the critical temperature are shown for comparison.

Fig. 2 The coefficients $F_{q}$ and $F_{\pi}$ appearing in eqs. (3.3), (3.6) and (3.12) which directly compare the rates for $q^{-} q^{+} l^{+} l^{-}$and $\pi^{+} \pi^{-} \rightarrow l^{+} l^{-}$. The $\rho$ and $\rho^{\prime}$ peaks are clearly evident.

Fig. 3 The dilepton mass distribution at central rapidities is shown for various combinations of initial and critical temperatures. Also shown is the Drell-Yan contribution which is expected to scale like $A^{4 / 3}$, and the measured low mass spectrum in pp collisions scaled by $A^{2 / 3}$.

Fig. 4 The dilepton rapidity density due to thermal emission divided by the square of the charged pion rapidity density reaches a plateau in the quark-gluon phase.

Fig. 5 The extinction of the vector meson peaks is more pronounced for larger values of the ratio $T_{0} / T_{c}$. 


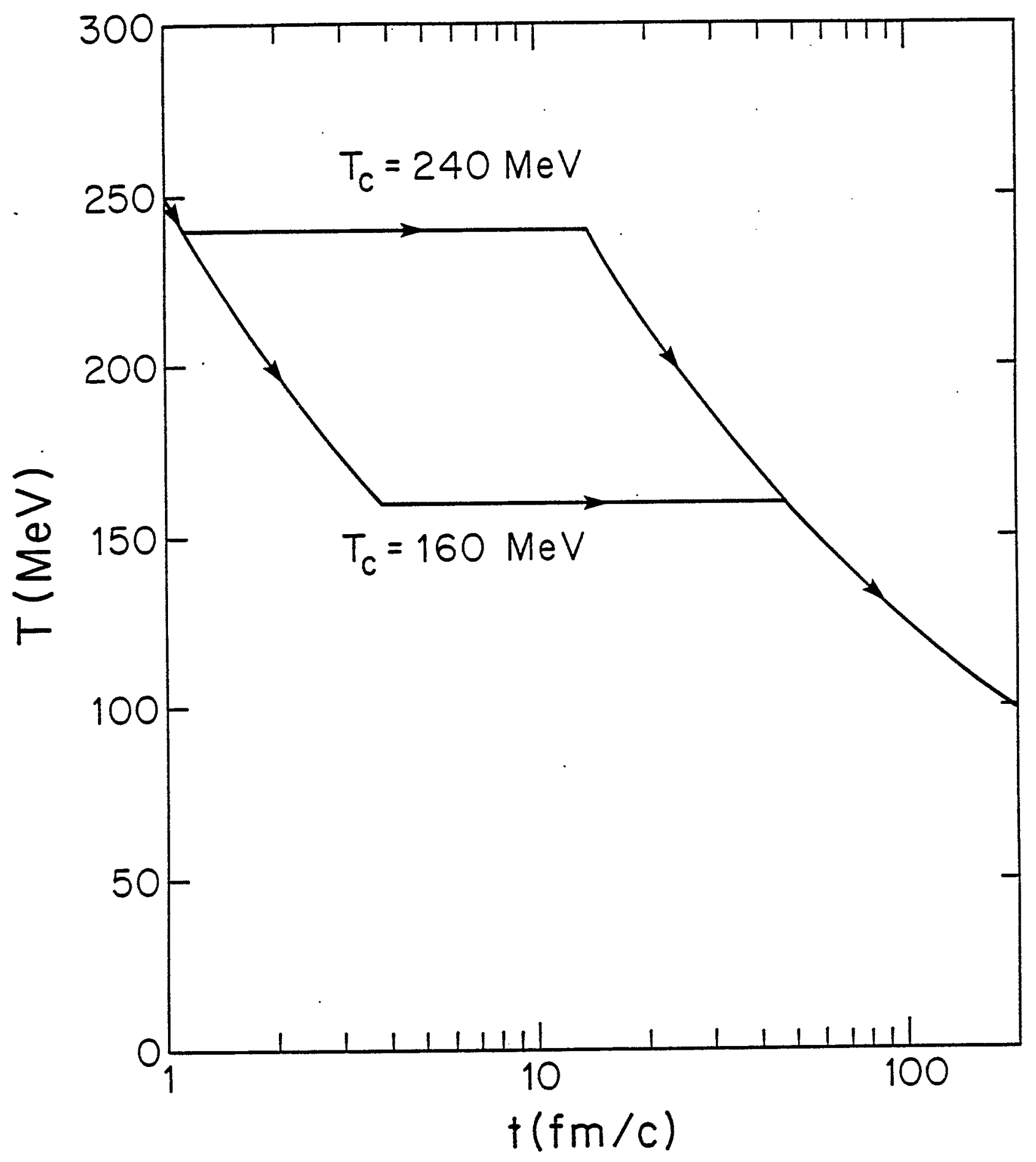

Fig. 1 


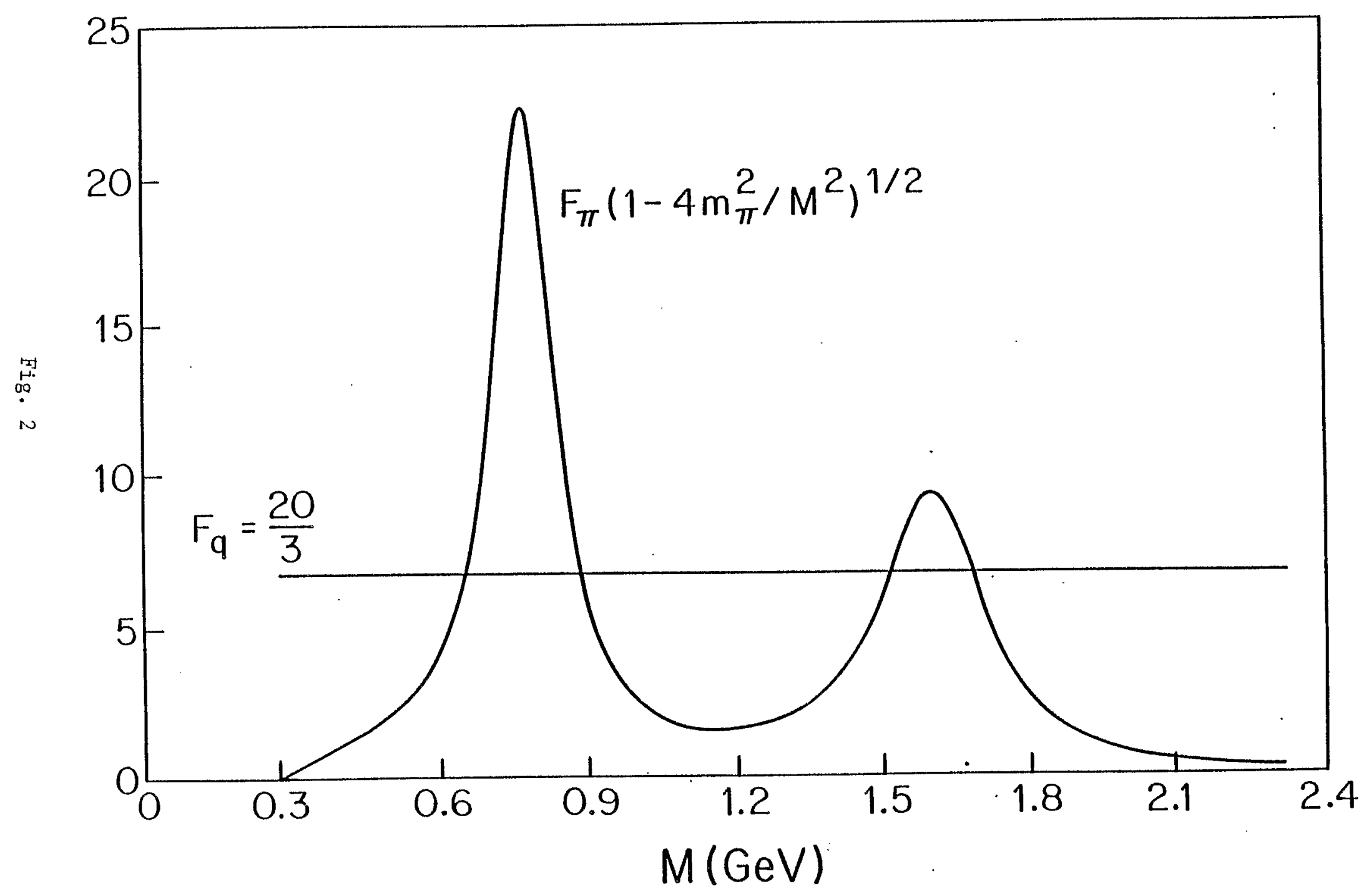




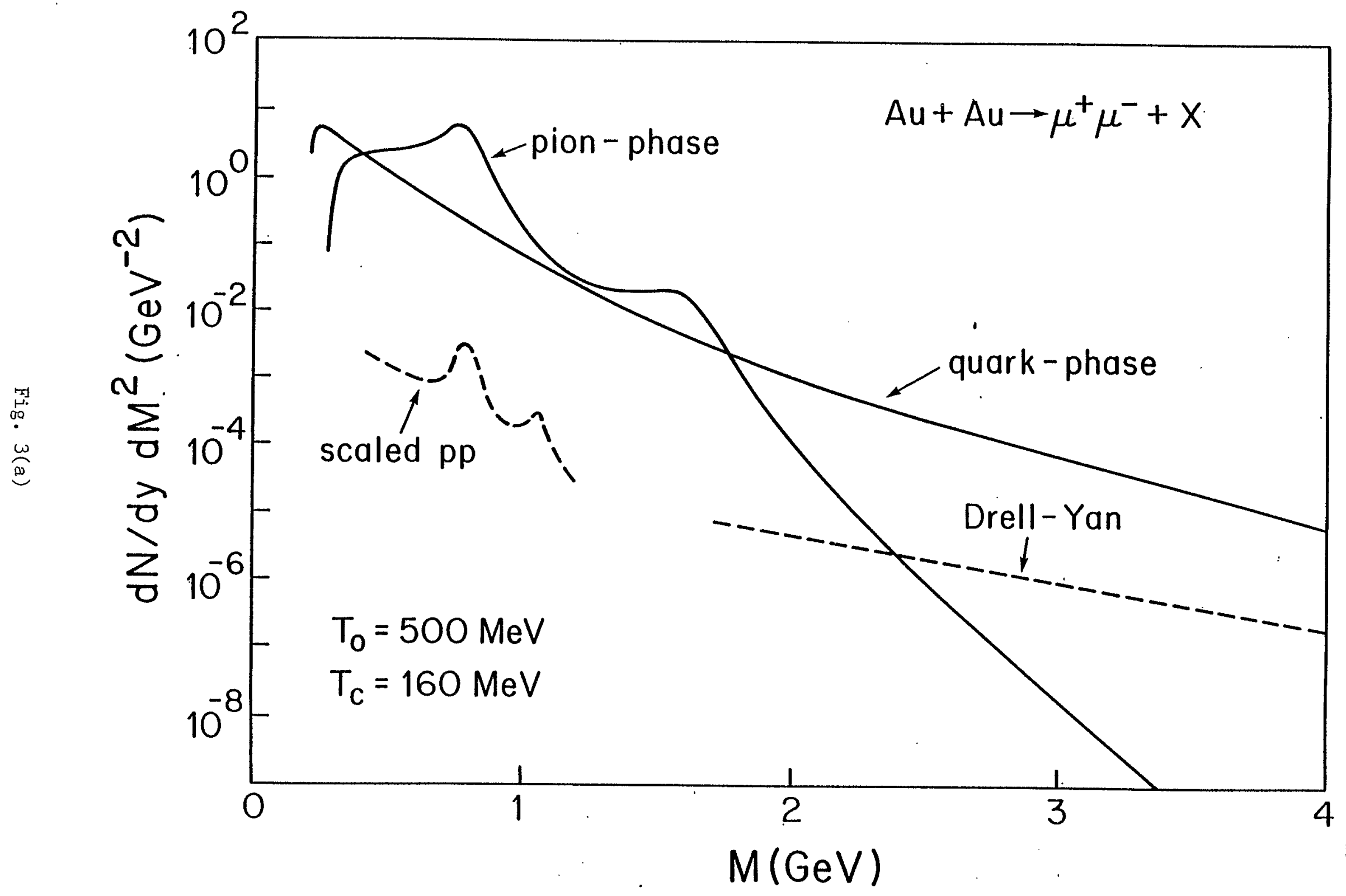




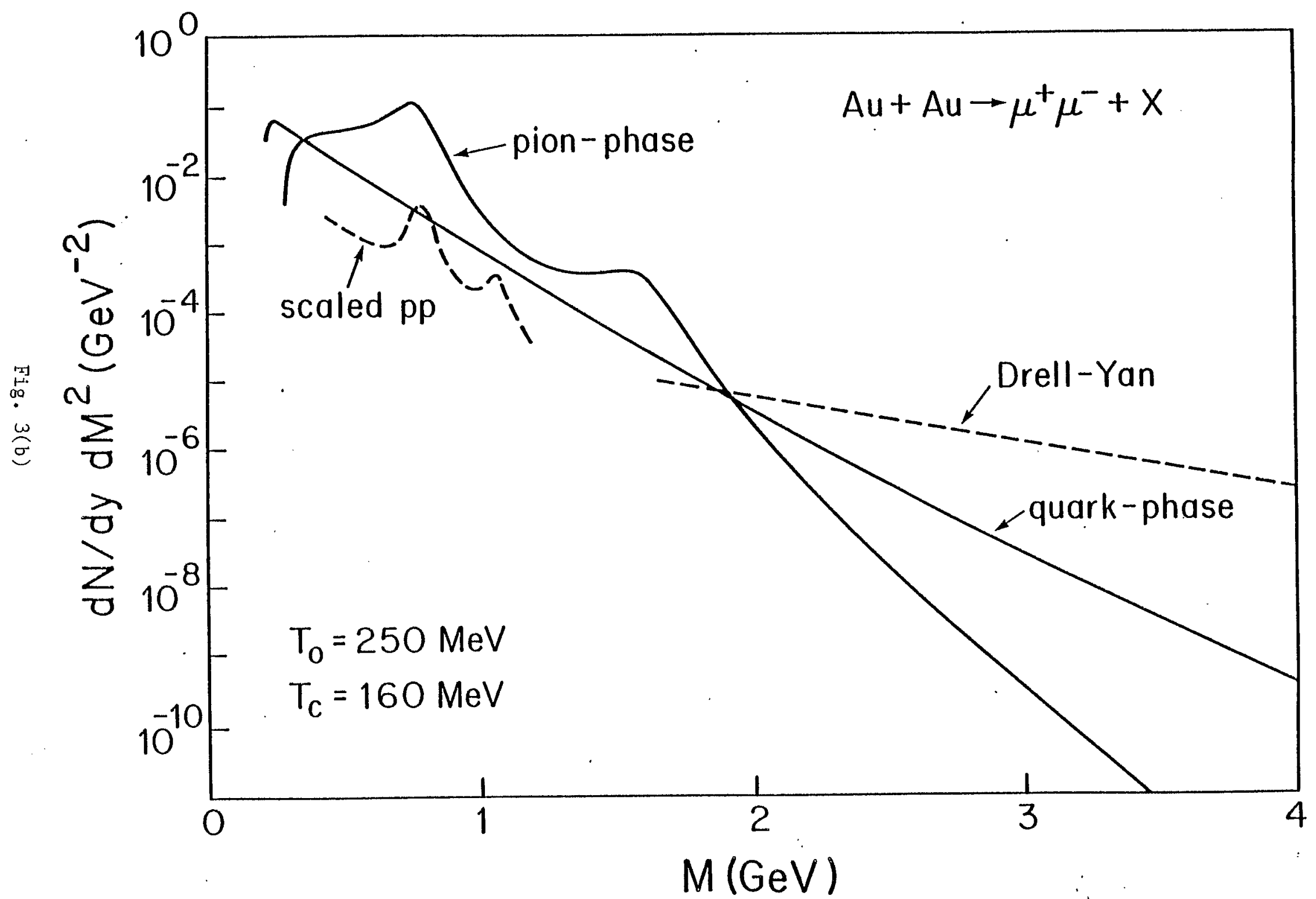




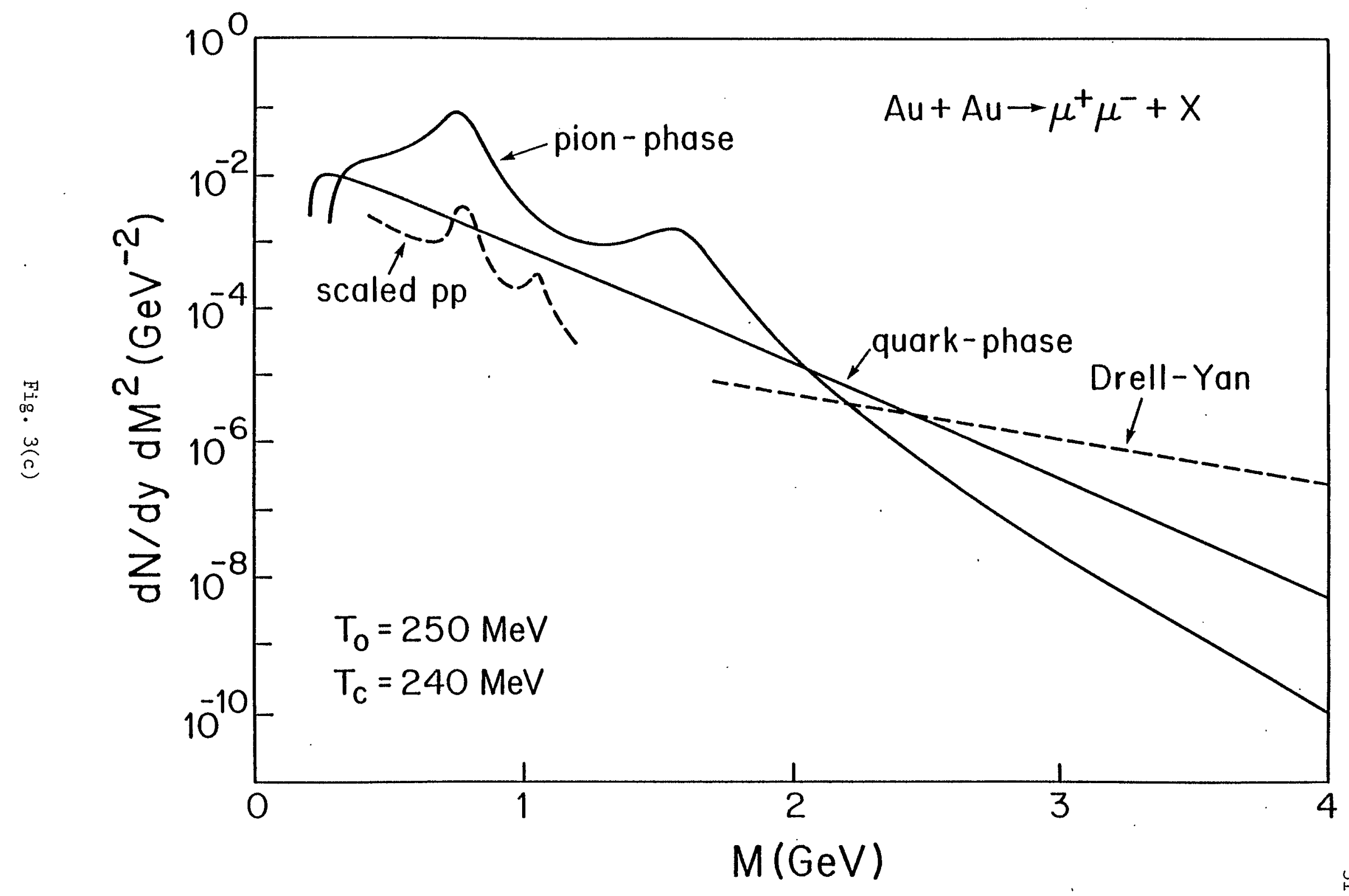




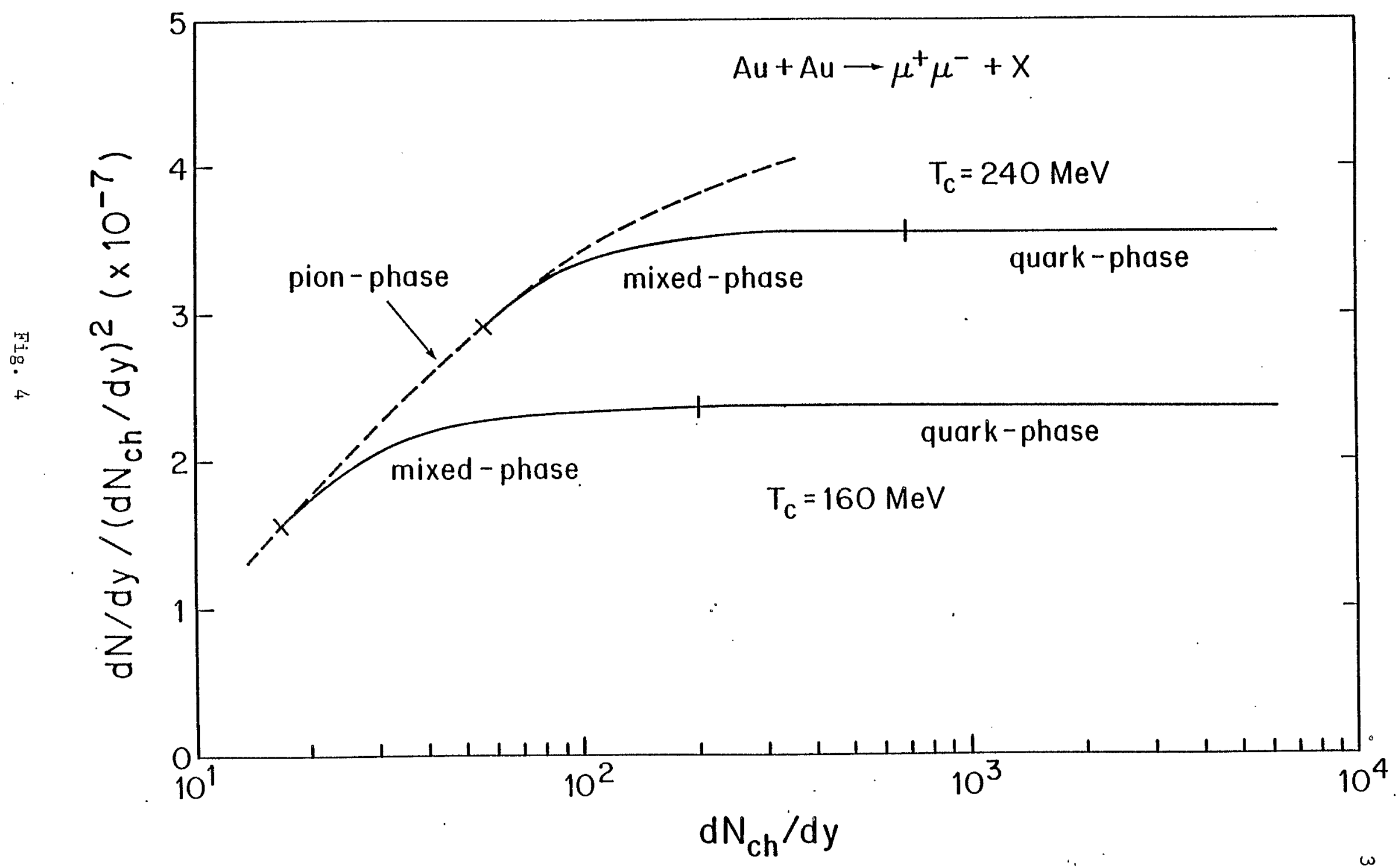




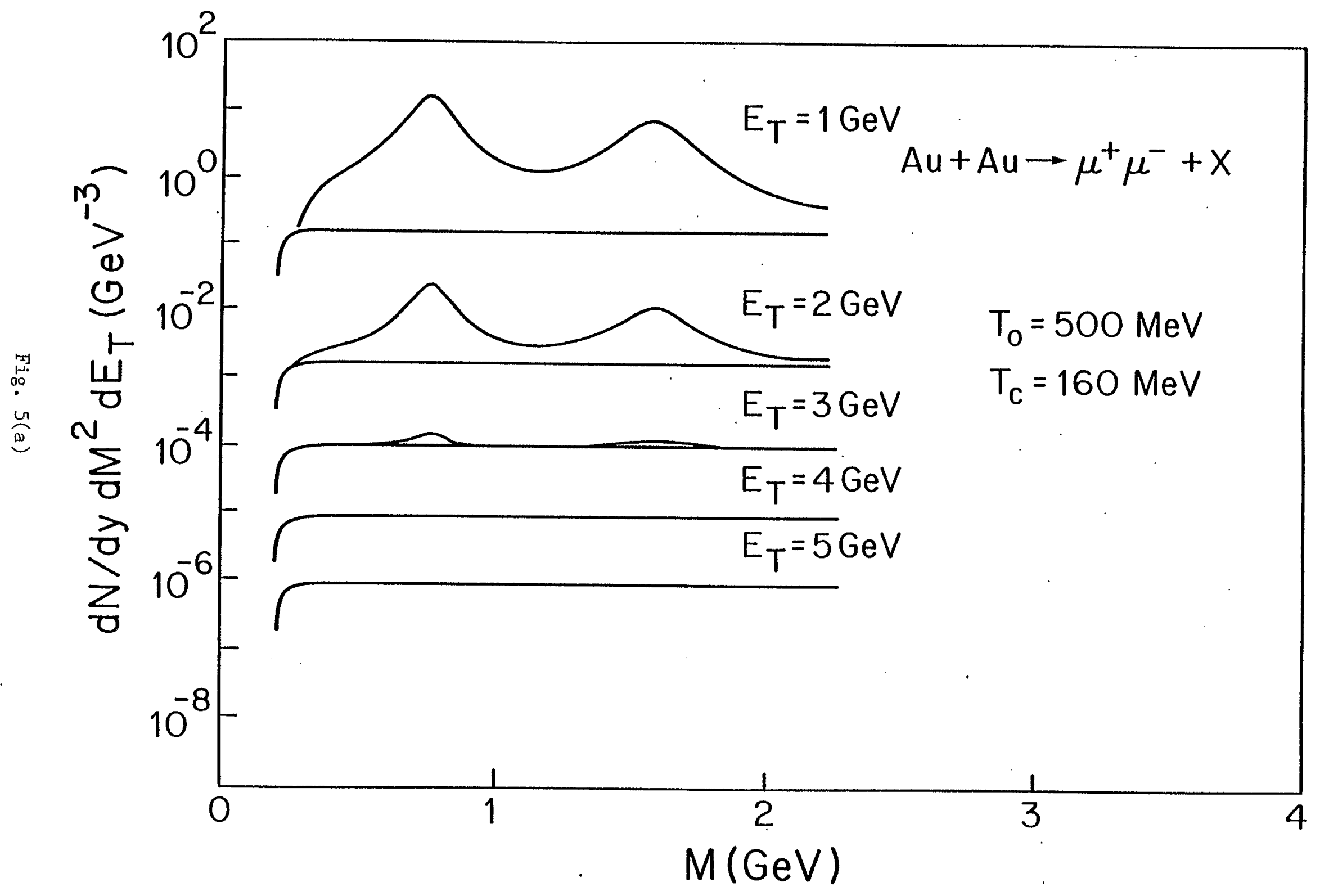




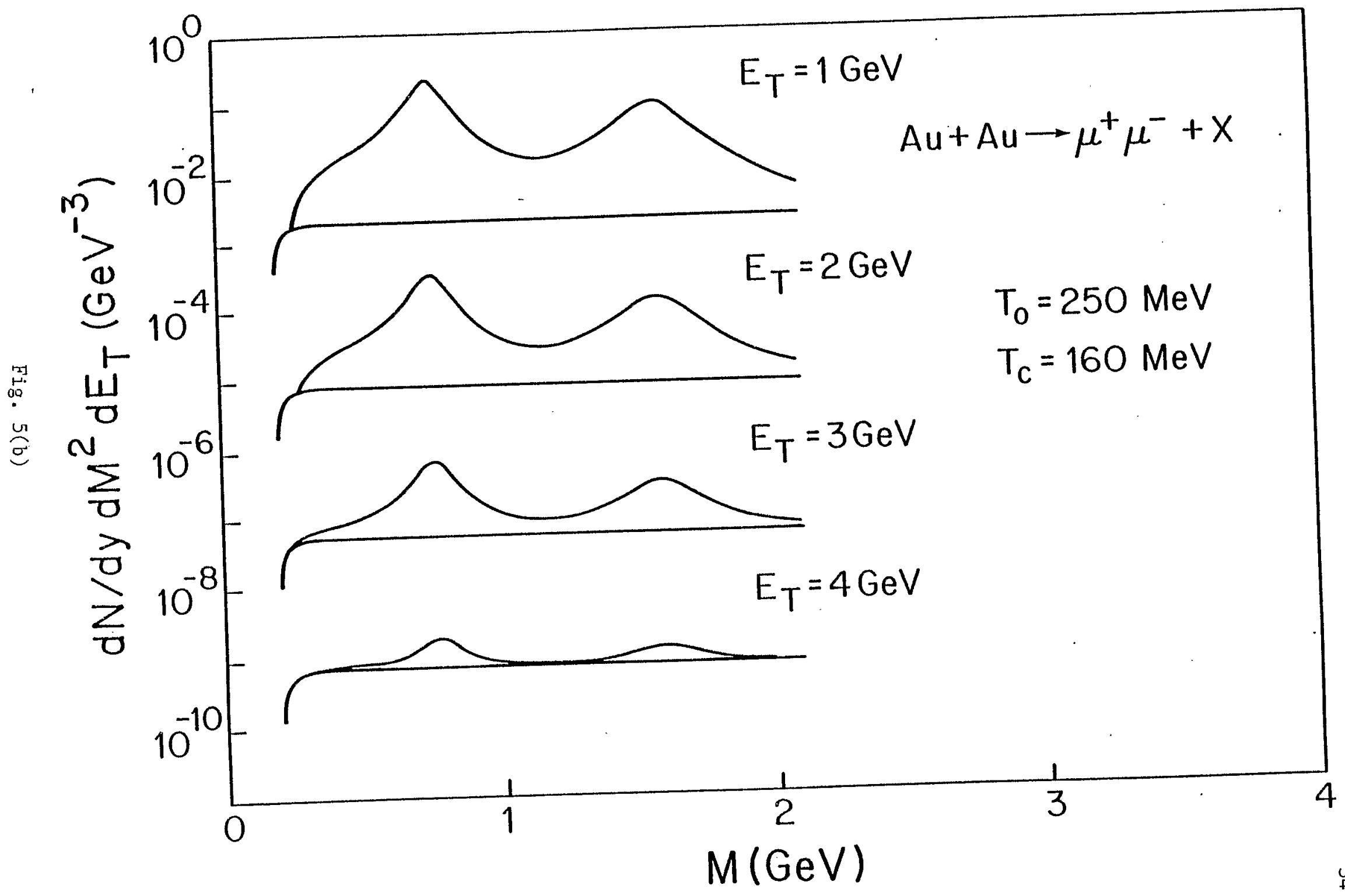




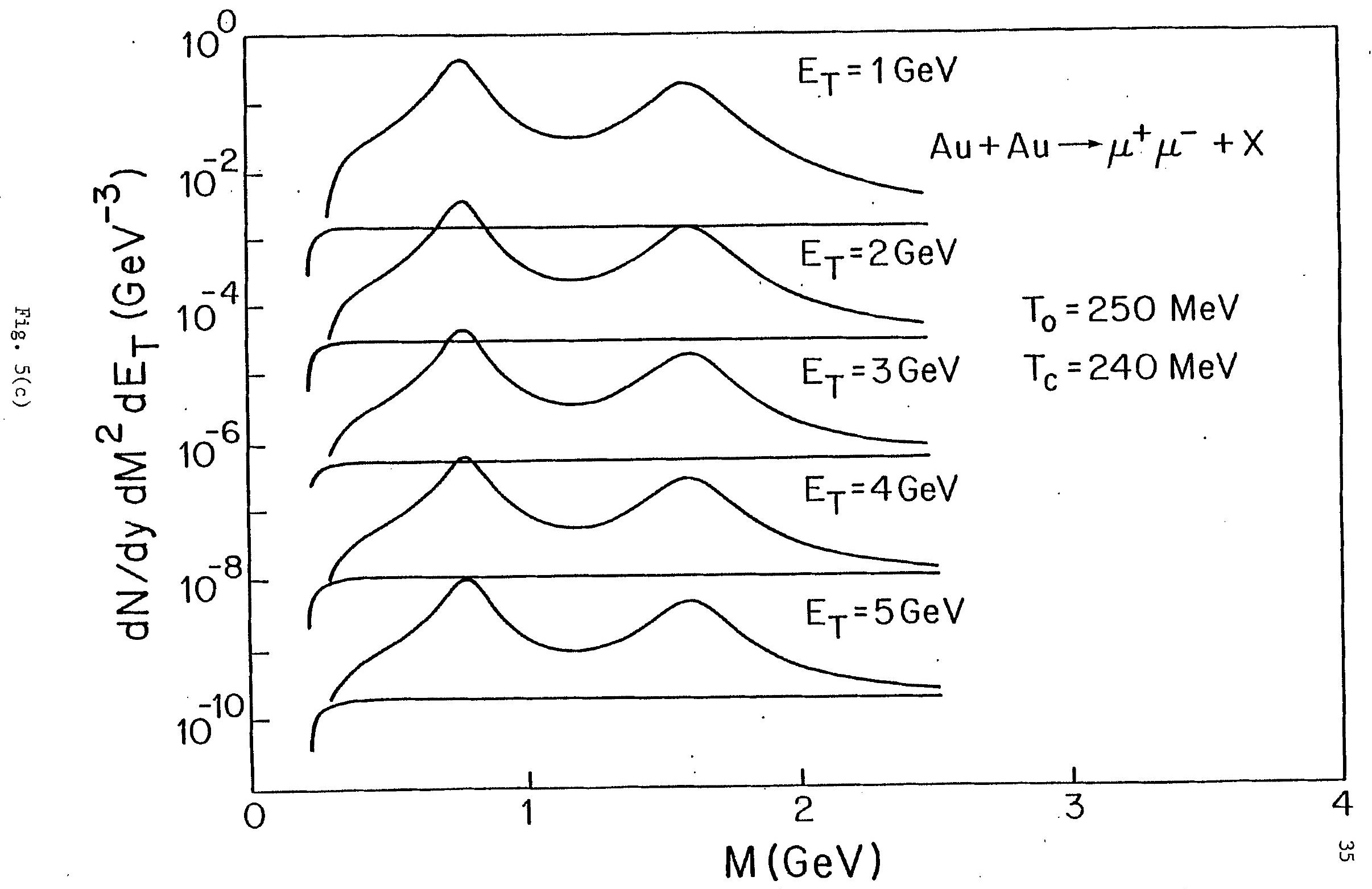

